\title{
Hysteroscopy findings and its correlation with latent endometrial tuberculosis in infertility
}

\author{
Subrat Kumar Mohakul • Venkata Radha Kumari Beela • \\ Purnima Tiru
}

Received: 18 May 2014 / Accepted: 8 October 2014 / Published online: 6 November 2014

(C) Springer-Verlag Berlin Heidelberg 2014

\begin{abstract}
In India, 5 to $18 \%$ of females attending infertility clinics are diagnosed to be suffering from genital tuberculosis. The present study was conducted to find out the prevalence of endometrial tuberculosis in infertility and its correlation with hysteroscopic changes. Patients attending infertility clinic with history of more than 2 years of unexplained infertility, failure to conceive in spite of successful ovulation induction in anovulatory infertility, and secondary infertility with a history of unexplained abortion or ectopic pregnancy were included in the study. In all the 105 cases, hysteroscopy was done, and the endometrium was subjected to DNA-PCR (polymerase chain reaction) testing for detection of Mycobacterium tuberculosis infection. Hysteroscopy features were compared in tuberculosis positive (39\%) and negative (61\%) cases for correlation. Tuberculosis was detected in $43.75 \%$ of ostial and periostial fibrosis, $48.48 \%$ of intrauterine fibrosis, and $66.67 \%$ of the irregular cavity surface. A complete 6-month course of antitubercular treatment was given to the tuberculosis positive cases among which $39 \%$ conceived without any additional treatment. The pregnancy rate of $64.7 \%$ in secondary infertility and $20.8 \%$ in primary infertility was very much promising. Tuberculosis is one of the major etiological factors in female infertility in developing countries. Preliminary assessment by hysteroscopy followed by PCR testing for Mycobacterium tuberculosis will detect it early. Subsequent antitubercular treatment may reverse the reproductive capability and prevent permanent damage to the female reproductive organs.
\end{abstract}

\footnotetext{
S. K. Mohakul $(\bowtie) \cdot$ V. R. K. Beela • P. Tiru

Visakha Steel General Hospital (RINL), Visakhapatnam, India

e-mail: skmohakulvizag@gmail.com

V. R. K. Beela

e-mail: bradhaaram@rediffmail.com

P. Tiru

e-mail: drpurnimatiru@vizagsteel.com
}

Keywords Hysteroscopy · Infertility · Latent endometrial tuberculosis $\cdot$ Female genital tuberculosis

\section{Introduction}

Genital tuberculosis (TB) in females is by no means uncommon, particularly in communities where pulmonary or other forms of extragenital TB are common. TB can affect any organ in the body, can exist without any clinical manifestation, and can recur. Female genital TB is typically understood as a disease of young women, with 80 to $90 \%$ of cases diagnosed in patients 20 to 40 years old, often during workup for infertility [1]. Genital tuberculosis in females is found in 0.75 to $1 \%$ of gynecological admissions in India, with considerable variation from place to place [2]. The disease is responsible for $5 \%$ of all female pelvic infections and occurs in $10 \%$ of cases of pulmonary tuberculosis [3]. It is estimated that a third of the world's population is infected with tuberculosis and that a new infection occurs every second [4]. Most of these infections are asymptomatic and may not cause disease. However, TB remains a major health problem in many developing countries, and in these areas, genital TB is responsible for a significant proportion of female infertility [5].

It is often a secondary complication as a result of the reactivation of a silent bacillemia, primarily from the lungs, affecting most commonly the fallopian tubes (92-100\%), endometrium (50\%), ovaries (10-30\%), cervix (5\%), and the vagina and vulva $(<1 \%)[2,6]$ but in some instances also from the kidney and intestines, etc. [7]. However, a few reports have found the endometrium to be the most commonly involved site [8,9]. Direct inoculation of Mycobacterium can also take place over the vulva or vagina during sexual intercourse with a partner suffering from tuberculosis of the genitalia [10]. Establishment of the true incidence and prevalence of female genital tuberculosis is difficult because asymptomatic latent cases predominate over symptomatic ones [11]. 
This silent invader of the genital tract tends to create diagnostic dilemmas because of varied clinical presentations and diverse findings on imaging and endoscopy. Although the histopathologic evidence of mycobacterial infection is highly indicative of genital $\mathrm{TB}$, its absence fails to exclude the infection. Identical lesions may also be seen in fungal and sarcoid diseases [12]. Similarly, culture methods, which have been considered a gold standard in proving genital TB, fail to exclude the infection. Furthermore, Mycobacterium is a temperamental bacillus that needs $4-5$ weeks to grow on Lowenstein-Jensen (LJ) media and 2 weeks time to grow on radiometric BACTEC media [13]. The minimum Mycobacterium concentration at which histopathologic evidence appears is 10,000 bacilli/ml. For positive cultures, the required concentrations are 1,000 bacilli $/ \mathrm{ml}$ for $\mathrm{LJ}$ and 10 $100 \mathrm{bacilli} / \mathrm{ml}$ for BACTEC media. But by utilizing DNAPCR (polymerase chain reaction) technique with high sensitivity of $96.4 \%$ and specificity of $100 \%$, tuberculosis can be detected in the concentration as low as 10 bacilli/ml [14].

Therefore, the present study was conducted to identify the tubercular infection of the endometrium by TB-PCR analysis and correlate it with hysteroscopy features and posttreatment outcomes.

\section{Materials and methods}

This prospective study was conducted in Vishakha Steel General Hospital between November 2008 and December 2011.

\section{Inclusion criteria}

The couples presenting to the outpatient department for treatment of infertility meeting the following criteria were included in the study:

1) Couples with more than 2 years of unexplained infertility where all investigations were within normal limits.

2) Couples with diagnosed anovulatory infertility who failed to conceive even after 6 cycles of successful ovulation induction.

3) Couples with secondary infertility following a history of unexplained abortion or ectopic pregnancy.

4) Primary or secondary infertility with hypomenorrhea of the female partner (scanty flow defined by menstrual loss of 2 days or less).

\section{Exclusion criteria}

Couples with severe male factor or bilateral tubal damage were excluded from the study.
In all the cases, a detailed history was taken followed by clinical examination and basic hematological investigations. Specialized infertility investigations like hormonal assay, thyroid profile, follicular study, tubal factor assessment, including laparoscopy were performed wherever indicated before including in the study. Hysteroscopy with simultaneous TBPCR testing of the endometrium was done for all the cases who qualified as per the inclusion criteria.

During diagnostic hysteroscopy, normal saline was used as the distension medium. A continuous flow double sheath hysteroscope was used to visualize the uterine cavity. The uterine wall and fundus were looked at for fibrosis, adhesion bands, ridges, and synechiae, indicating intrauterine fibrosis (Figs. 1, 2, 3, 4, and 5). Internal tubal ostia were checked for stenosis, a pinhole opening, blocks, and periostial fibrosis (Fig. 6). The surface of the uterine cavity was examined for irregularity (Fig. 7) or any other abnormality (Fig. 8). After the completion of hysteroscopy, a sample from the endometrium was collected by sharp curettage and stored in normal saline for TB-PCR testing.

Mycobacterium DNA was extracted from endometrial tissue using Qiagen DNA Mini Kit. DNA binds specifically to the silica-gel membrane while contaminants pass through. PCR inhibitors such as divalent cations and proteins are completely removed in two efficient wash steps, leaving purified DNA. The protocol mentioned by the manufacturer was exactly followed to obtain a final elute of $200 \mu \mathrm{l}$ which was then subjected to real time PCR.

Oligonucleotides-designated Sp1 (5_-ACCTCCTTTCTA AGGAGCACC-3_) and Sp2 (5_-GATGCTCG CAACCA CTATCCA-3_) were used to amplify an approximately 220bp fragment of the ITS (16S-23S rDNA internal transcribed spacer) sequence (European Bioinformatics Institute accession number L15623) from Mycobacterium. Amplified product was detected by using modified specific fluorescent probes. In order to specifically identify mycobacterium tuberculosis (MTB), paired fluorogenic hybridization probes were designed to recognize a region in the ITS fragment [15].

For each sample, $10 \mu \mathrm{l}$ of template DNA was incorporated into a 50- $\mu$ PCR containing the amplification oligonucleotides and MTB-ITS hybridization probes using the QuantiTect Probe kit (Qiagen). The optimized PCR protocol included an initial denaturation step at $95^{\circ} \mathrm{C}$ for $30 \mathrm{~s}$ and was followed by a three-step PCR cycle $95^{\circ} \mathrm{C}$ for $30 \mathrm{~s}, 59^{\circ} \mathrm{C}$ for $5 \mathrm{~s}$, and $72^{\circ} \mathrm{C}$ for $30 \mathrm{~s}$ for 40 cycles. Fluorescence measurements are made in every cycle. The threshold cycle $(\mathrm{Ct})$ value is the cycle at which there is a significant increase in fluorescence, and this value is associated with an exponential growth of PCR product during the log-linear phase. Positive and negative controls were used in each run. A melt curve analysis performed on the Rotor-Gene 3000 confirmed the presence of ITS fragment amplification specific to mycobacterium tuberculosis complex (MTC) in all specimens. 
Fig. 1 Normal hysteroscopy with normal left ostium and

endometrial glands

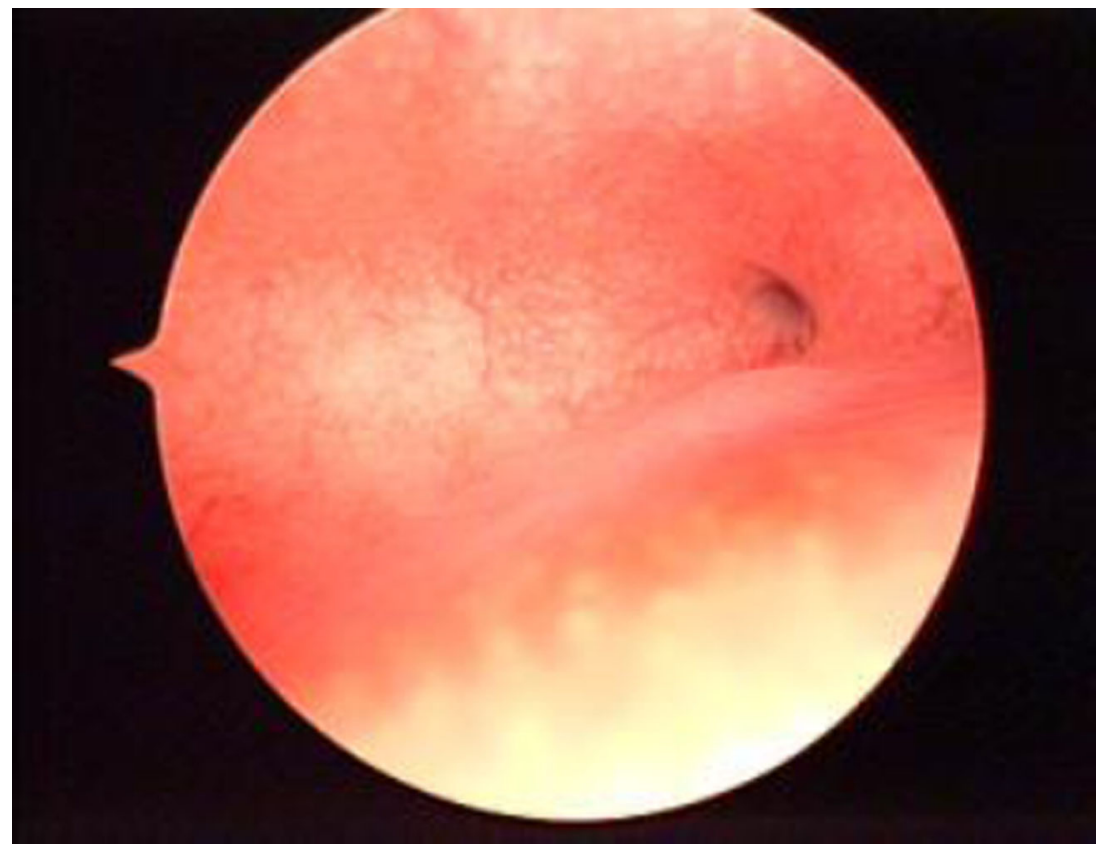

The hysteroscopy findings were analyzed and correlated with TB-PCR results. Those cases were found to be positive for TB-PCR were given a 6-month course of antitubercular treatment (ATT). The ATT consists of a four-drug regimen, i.e., rifampicin, isoniazid, ethambutol, and pyrazinamide for 2 months, followed by rifampicin and isoniazid for 4 months. Liver function test (LFT) was performed before starting ATT and repeated every month. Those showing common adverse effects like nausea and vomiting were managed with domperidone and ranitidine. Complaints of neuropathic symptoms were managed by a pyridoxine supplement.

Fig. 2 Fundal fibrosis

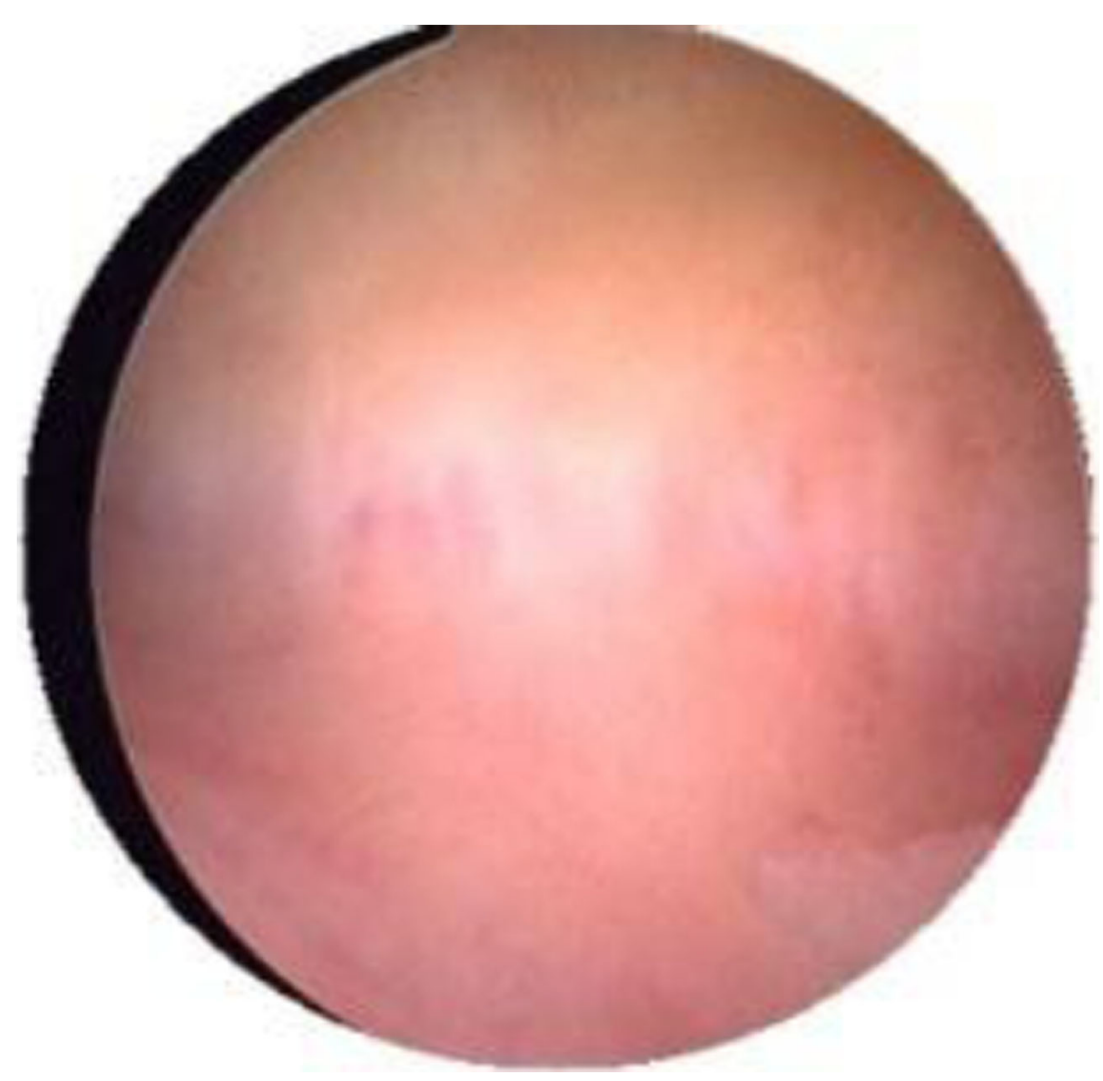


Fig. 3 Fibrotic band

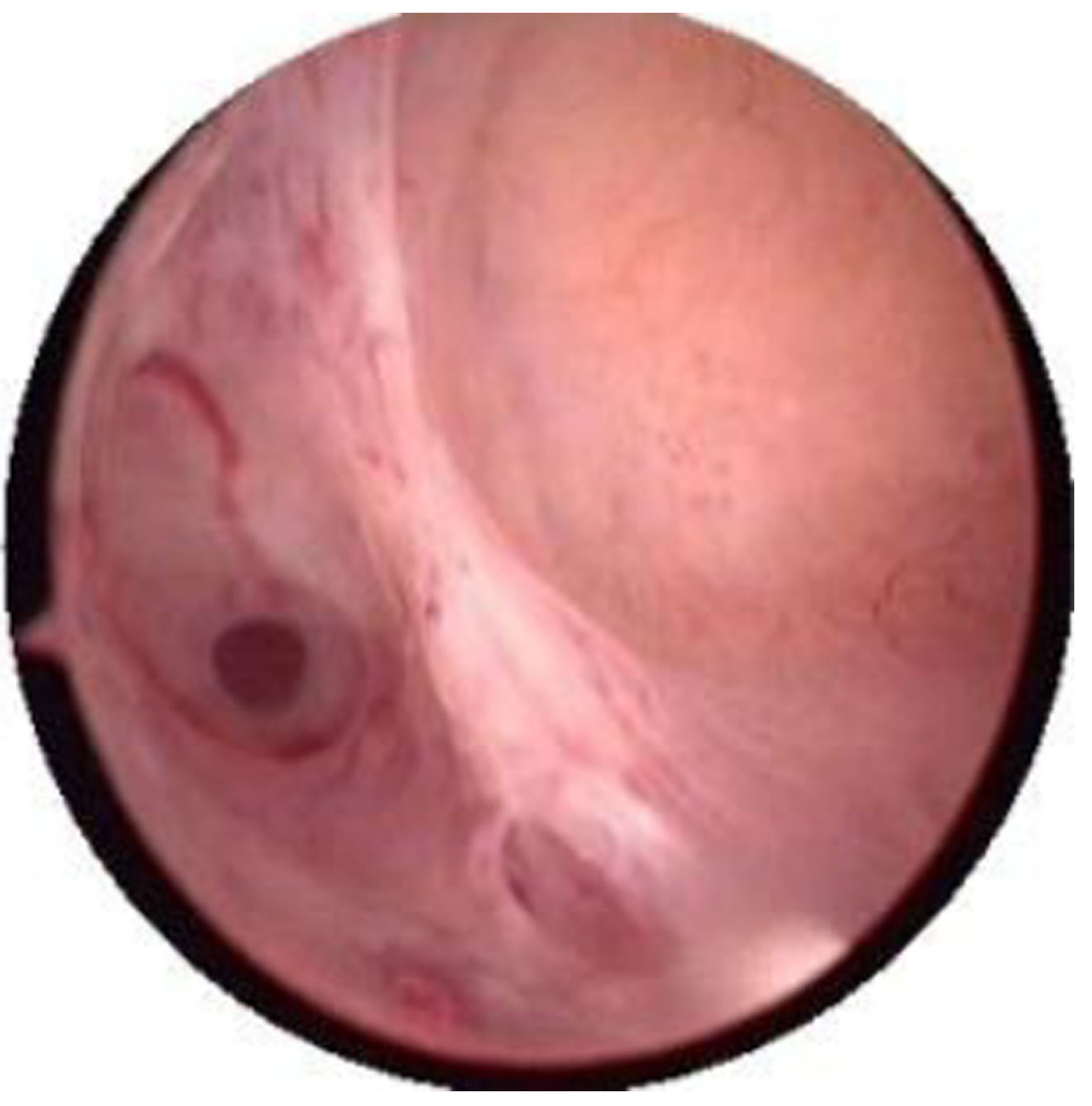

Fig. 4 Fibrotic ridge

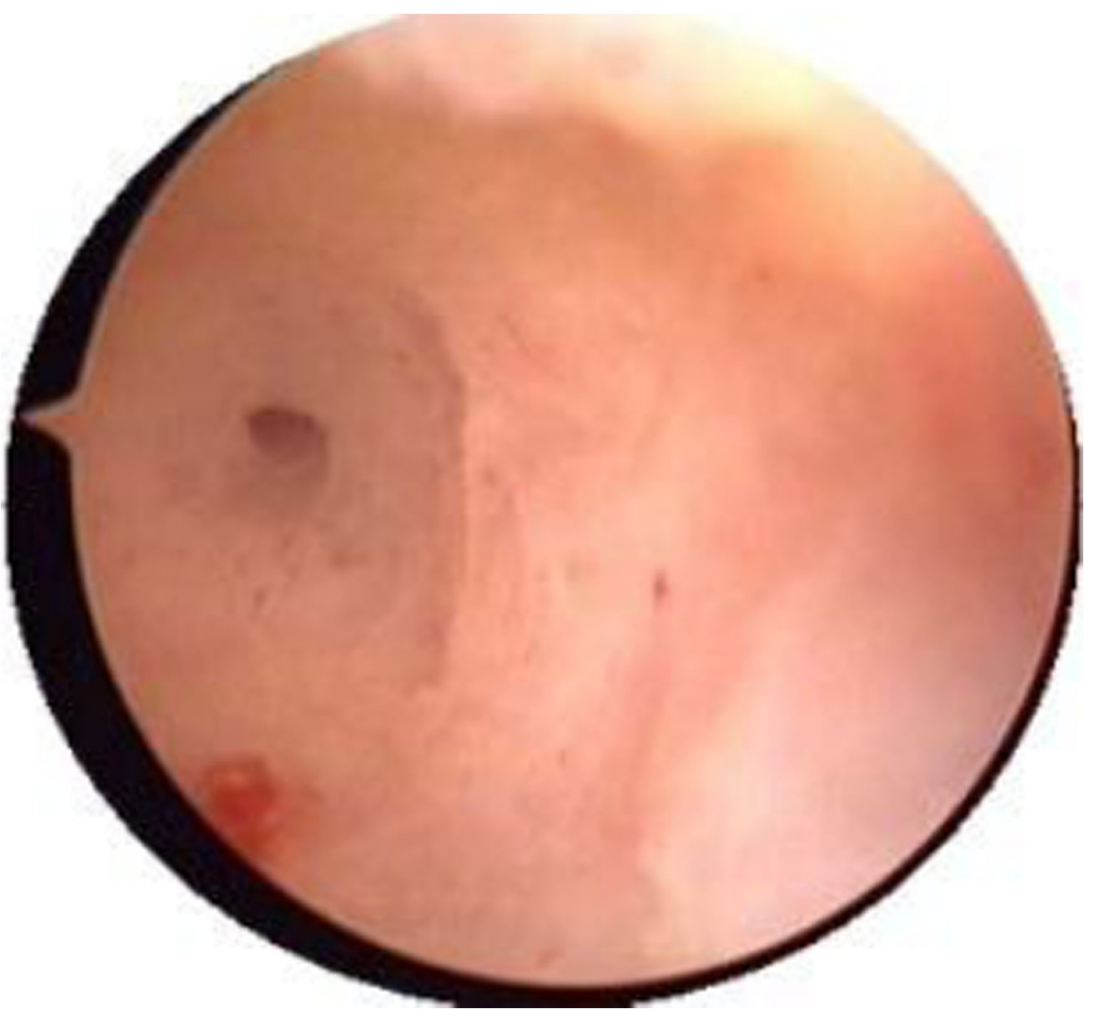


Fig. 5 Intrauterine synechiae

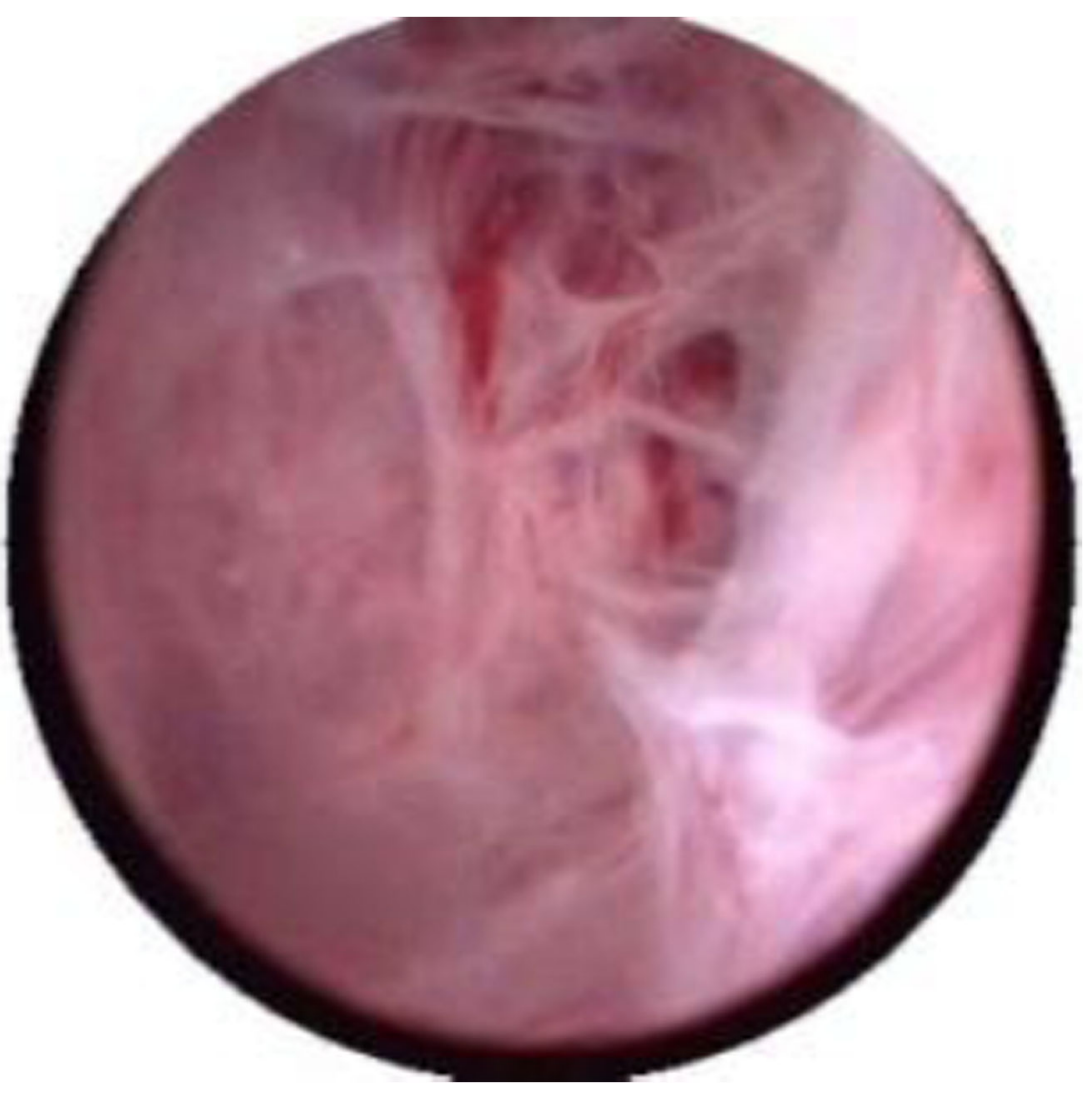

\section{Results}

In this study, a total number of 105 patients with complaints of infertility, meeting the inclusion criteria, were analyzed for the correlation of TB-PCR results with hysteroscopy findings. The treatment outcomes in terms of pregnancy rates were also analyzed. Majority ( $38 \%$ ) of cases presented between 26 and 30 years of age followed by the age group of 20 to 25 years

Fig. 6 Periostial fibrosis with pinhole left ostium

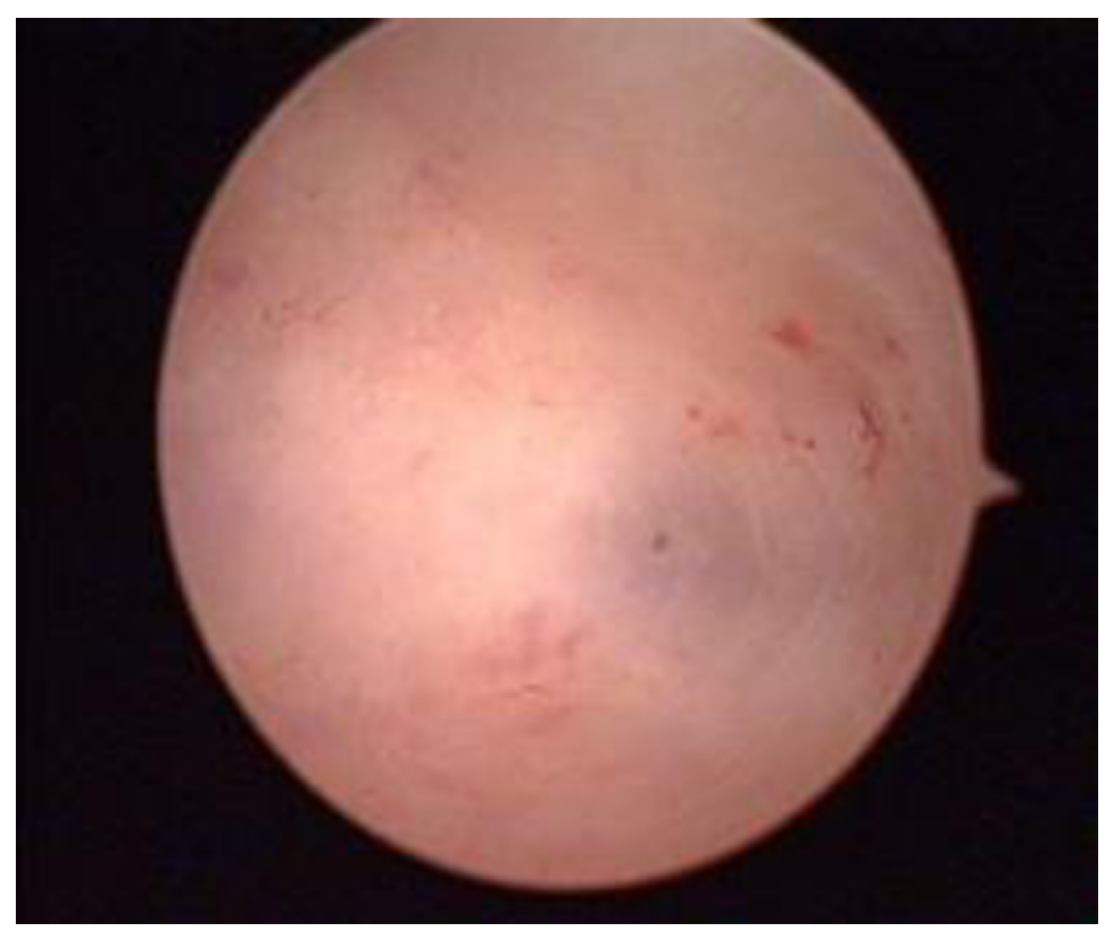


Fig. 7 Irregular endometrial surface

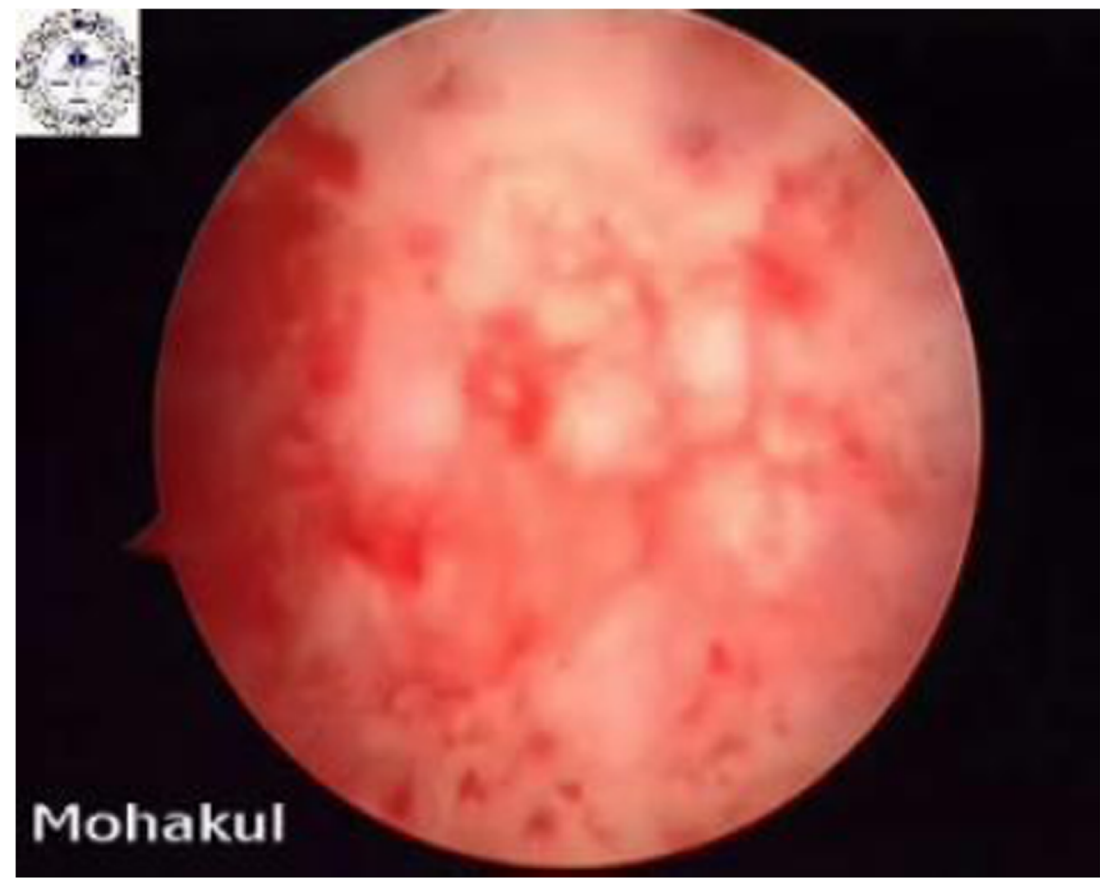

(35\%) (Table 1). Fifty-eight percent of the cases had primary infertility, and $42 \%$ had secondary infertility. In $39 \%$ of cases, the presence of Mycobacterium tuberculosis was detected in the endometrial tissue (TB-PCR positive) (Table 2).

Analyzing the hysteroscopy findings, it was found that among patients showing ostial and periostial fibrotic changes, there was $43.75 \%$ of positive TB-PCR cases. Intrauterine fibrosis was associated with positive TB-PCR in $48.48 \%$ of cases. Patients having irregular cavity wall was found to have $66.67 \%$ of positive TB-PCR rate. At last, there was a single case of spotted endometrium and the same was positive for TB-PCR (Table 3).

Fig. 8 Spotted endometrium

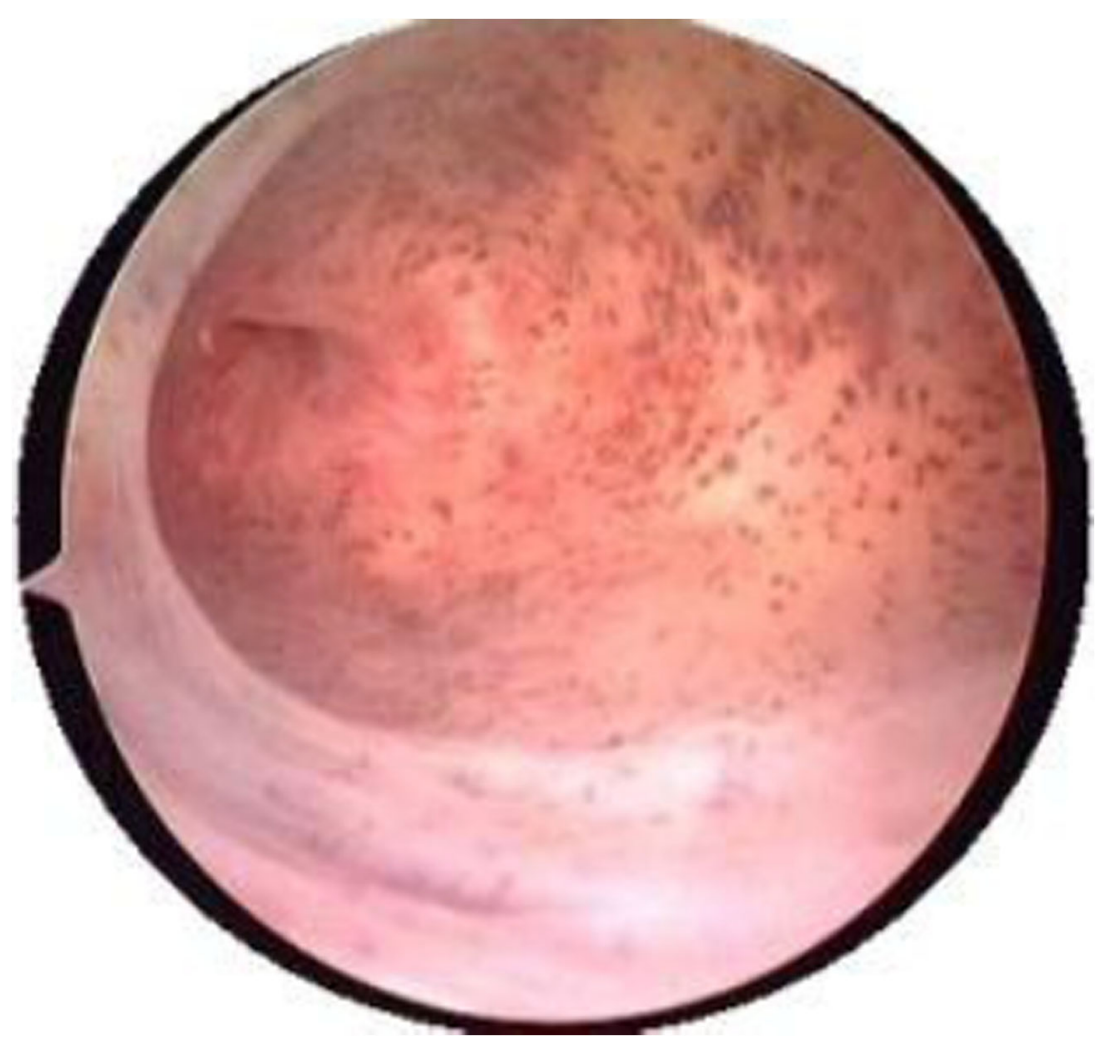


Table 1 Age distribution

\begin{tabular}{lll}
\hline Age (years) & No. of cases & Percentage (\%) \\
\hline $20-25$ & 37 & 35 \\
$26-30$ & 40 & 38 \\
$31-35$ & 13 & 13 \\
$>35$ & 15 & 14 \\
\hline
\end{tabular}

Out of all TB PCR positive cases, $39 \%$ conceived with antitubercular treatment only without any surgical intervention (Table 2). A successful conception rate of $64.7 \%$ was noted in secondary infertility whereas it was $20.8 \%$ in primary infertility (Table 2 ).

The most common side effects of ATT were nausea and vomiting which was managed with domperidone and ranitidine. Neuralgia and tingling sensation were complained by very few patients and were managed successfully by $100 \mathrm{mg}$ of pyridoxine supplementation per day. Abnormal liver function test with icterus and high values of liver enzymes leading to abandonment of treatment was observed in two patients during the initial 2 months of treatment and were hence excluded from the study.

\section{Discussion}

Female genital tuberculosis is a rare disease in developed countries but is a frequent cause of chronic pelvic inflammatory disease and infertility in underdeveloped and developing countries. Various studies show genital TB as a cause of infertility in $1-18 \%$ of the cases, around $1 \%$ in the developed countries and $18 \%$ in India $[1,5]$.

Generally, Mycobacterium gains access through respiratory passage in lungs and then disseminates to different organs via the lymphatic drainage and blood circulation. The female genital tract is also an important site in this dissemination process. Once the immunity to $M$. tuberculosis contains and overrides the growth of small number of disseminated TB bacilli after primary infection, the infection may remain silent throughout life until lowered immunity or overwhelming reinfection precipitates the disease [16]. Increased circulation and hormone dependence of the female genital organs after

Table 2 Treatment results

\begin{tabular}{llll}
\hline $\begin{array}{l}\text { Types of } \\
\text { infertility }\end{array}$ & No. of cases & $\begin{array}{l}\text { TB-PCR } \\
\text { positive cases }\end{array}$ & $\begin{array}{l}\text { Conception rate among PCR } \\
\text { positive cases after ATT }\end{array}$ \\
\hline Primary & $61(58 \%)$ & $24(39.3 \%)$ & $5(20.8 \%)$ \\
Secondary & $44(42 \%)$ & $\begin{array}{l}17(38.63 \%) \\
41(39 \%)\end{array}$ & $16(64.7 \%)$ \\
Total & 105 & $41(39 \%)$ \\
\hline
\end{tabular}

${ }^{*} p=0.002$
Table 3 Hysteroscopy findings and its correlation with TB-PCR

\begin{tabular}{lll}
\hline Hysteroscopic findings & No. of patients & TB-PCR positive \\
\hline Ostial and periostial fibrosis & $32(30.48 \%)$ & $14(43.75 \%)$ \\
Intrauterine fibrosis & $66(62.86 \%)$ & $32(48.48 \%)$ \\
Irregular cavity wall & $3(2.86 \%)$ & $2(66.67 \%)$ \\
Spotted endometrium & $1(0.95 \%)$ & $1(100 \%)$ \\
Normal-looking cavity & $3(2.86 \%)$ & 0 \\
Total no. of cases & 105 & $41(39 \%)$ \\
\hline
\end{tabular}

sexual maturity may in part explain why the genital system is vulnerable to this infection after puberty [17]. The tuberculous process generally is localized to the endometrium, being most extensive in the fundus and decreases toward the cervix. The myometrium is not usually involved. In premenopausal patients, much of the infected tissue is shed during the menstruation, only to have the endometrium reinfected from the tubes with each cycle.

In genital $\mathrm{TB}$, there is a high incidence of involvement of the endometrium. Schaefer reported an incidence of 50-60\% [1], Onuigbo, an incidence of $60 \%$ [18], and NogalesOrtiz and colleagues, an incidence of $79 \%$ [8], whereas Sutherland estimated $90 \%$ involvement of the endometrium in genital TB [9].

In endometrial tuberculosis, infertility is due to functionally altered endometrium or associated tuberculous salpingitis [19]. Tubercular infection greatly suppresses the sensitivity of the endometrium to ovarian hormones leading to deficient secretory phase and defective secretion of glycogen [20]. This results in defective implantation of an ovum, leading to infertility [21]. Therefore, if the infection could be diagnosed and treated early enough before permanent damage to the genital organs ensues, it may be possible to regain the reproductive capability.

Traditionally, the laboratory diagnosis of TB depends on demonstration of the causative organism, by acid-fast staining and/or growth of the organism on Lowenstein-Jensen (LJ) medium. The diagnosis of tubercular involvement of the endometrium is difficult because it is a paucibacillary infection, and infected endometrium is shed off during menstruation. Culture methods fail to exclude the infection, and it needs 4-5 weeks to show growth on LJ media and 2 weeks time on radiometric BACTEC media. The minimum Mycobacterium concentration at which histopathological evidence appears is $10,000 \mathrm{bacilli} / \mathrm{ml}$. For positive culture, the required concentrations are 1,000 bacilli/ml for $\mathrm{LJ}$ and 10-100 bacilli/ml for BACTEC media [14]. Therefore, a more sensitive method is required for the early diagnosis of endometrial tuberculosis specifically in latent cases.

During the 1990s, nucleic acid amplification (NAA) techniques evolved and dramatically altered the way in which we can detect and identify M. tuberculosis. NAA technique is a 
PCR-based test that amplifies M. tuberculosis DNA for the detection of $M$. tuberculosis from samples containing as low as $10 \mathrm{bacilli} / \mathrm{ml}$ [14]. Due to this reason, DNA-PCR techniques were utilized to identify $M$. tuberculosis directly from the clinical specimens, and the results were available in a day or two with high sensitivity ( $96.4 \%$ ) and high specificity (100\%) [22].

In our study, we tried to correlate the hysteroscopic changes to the presence of Mycobacterium in the endometrial tissue in cases of infertility where there were no other obvious causes detected. As we can understand that the major physical changes to the endometrium takes a long time to appear because of the repeated shedding, we have concentrated on the subtle as well as gross hysteroscopic changes that may suggest any low-grade inflammation. Therefore, minimal changes like pinhole ostium, periostial fibrosis and gross changes like fundal fibrosis, intrauterine fibrotic bands, and blocked ostia were all taken into consideration for assessing the possible involvement of the endometrium.

Because of targeting a very selective group of population and including subtle changes as abnormal, we found only $2.86 \%$ of normal-looking endometrium. In our study, ostial and periostial fibrosis was associated with positive TB-PCR in $43.75 \%$ and intrauterine fibrosis was associated with positive TB-PCR in $48.48 \%$ of cases. This finding strongly suggests looking for the presence of M. tuberculosis in such types of hysteroscopic pictures in infertility. Although irregular cavity surface and spotted endometrium were associated with 66.67 and $100 \%$ of positive TB-PCR, respectively, the number of patients was too minimal to derive any conclusion (Table 2). Baxi et al. reported $44.44 \%$ positive TB-PCR in ostial fibrosis and $50 \%$ positive TB-PCR in intrauterine fibrosis [23]. These reports were comparable to our study $(p=0.47)$. The overall incidence of $32.18 \%$ reported by Baxi et al. is also in close agreement with our report of $39 \%$ (Table 4).

Out of all TB-PCR positive cases in our study, $39 \%$ conceived with antitubercular treatment only, which is very a encouraging result. There was a drastic difference in conception rate between primary and secondary infertility. The conception rate of $64.7 \%$ in secondary infertility as against $20.8 \%$ of that in primary infertility $(p=0.002)$ indicates a higher singular contributory role of tuberculosis toward infertility in the first group (Table 2). The lower pregnancy rate in

Table 4 Comparison with the other study

\begin{tabular}{lll}
\hline Comparison with the other study & Our study & Baxi et al. study [23] \\
\hline Total & $N=105$ & $N=174$ \\
Ostial fibrosis & $43.75 \%$ & $44.44 \%$ \\
Intrauterine fibrosis & $48.48 \%$ & $50 \%$ \\
Overall total PCR positive & $39 \%$ & $32.18 \%$ \\
\hline
\end{tabular}

$* p=0.47$ primary infertility may indicate additional contributing factors apart from this acquired disease. Hence, subsequent IVF could be an ideal option for those who could not conceive after ATT.

\section{Conclusion}

Genital tuberculosis leading to tuberculous endometritis is one of the most intractable causes of infertility. Early diagnosis in the latent phase is possible by detecting the subtle hysteroscopic changes, supplemented by TB-PCR testing. A subsequent complete course of ATT may prevent the development of overt genital tuberculosis and reverse the reproductive capability. The results are very encouraging and more apparent in secondary than in primary infertility. Therefore, a largescale study is recommended to establish the exact role of latent endometrial tuberculosis in infertility in developing countries where tuberculosis is endemic.

Conflict of interest Subrat Kumar Mohakul, Venkata Radha Kumari Bella, and Purnima Tiru declare that they have no conflict of interest.

Informed Consent All procedures followed were in accordance with the ethical standards of the responsible committee on human experimentation (institutional and national) and with the Helsinki Declaration of 1975 , as revised in 2000. Informed consent was obtained from all patients for being included in the study.

\section{References}

1. Schaefer G (1976) Female genital tuberculosis. Clin Obstet Gynecol 19:223

2. Varma TR (1991) Genital tuberculosis and subsequent fertility. Int J Gynaecol Obstet 35:1-11

3. Dawn CS (1998) Pelvic infections. In: Dawn CS 9th ed. Textbook of gynaecology and contraception: 9th ed. Calcutta: Arati Dawn p 321

4. Bartlett JG (2007) Tuberculosis and HIV infection: partners in human tragedy. J Inf Dis 196:S124-S125

5. Muir DG, Belsey MA (1980) Pelvic inflammatory disease and its consequences in the developing world. Am J Obstet Gynecol 138: 913-928

6. Arora VK, Johri A, Arora R et al (1994) Tuberculosis of the vagina in a HIV seropositive case. Tuber Lung Dis 75:239-240

7. Simon HB, Weinstein AJ, Pasternak MS et al (1977) Genitourinary tuberculosis: clinical features in a general hospital population. Am J Med 63:410-420

8. Nogales-Ortiz F, Tarancion I, Nogales FF Jr (1979) The pathology of female genital tuberculosis: a 31-year study of 1436 cases. Obstet Gynecol 53:422

9. Sutherland AM (1960) Genital tuberculosis in women. Am J Obstet Gynecol 79:486

10. Richards MJ, Angus D (1998) Possible sexual transmission of genitourinary tuberculosis. Int J Tuberc Lung Dis 2:439

11. Rom W, Garay S (1996) Tuberculosis, 1st edn. Little Brown, New York

12. Krishna UR, Sheth SS, Motashaw ND (1979) Place of laparoscopy in pelvic inflammatory disease. J Obstet Gynaecol India 29(3):505-510 
13. Katoch VM (2004) Newer diagnostic techniques for tuberculosis. Indian J Med Res 120:418-428

14. Bhanu NV, Singh UB, Chakraborty M et al (2005) Improved diagnostic value of PCR in the diagnosis of female genital tuberculosis leading to infertility. J Med Microbiol 54(Pt10):927-931

15. Kraus G, Cleary T, Miller N, Seivright R, Young AK, Spruill G, Hnatyszyn HJ (2001) Rapid and specific detection of Mycobacterium tuberculosis using fluorogenic probes and real-time PCR. Mol Cell Probes 15:375-383

16. Arora VK, Gupta R, Arora R (2003) Female genital tuberculosisneed for more research. Indian J Tuberc 50:9

17. Choudhary NN (1996) Overview of tuberculosis of the female genital tract. J Indian Med Assoc 94:345-361
18. Onuigbo WIB (1979) Genital tuberculosis and reproductive function. J Reprod Med 21:249

19. Falk V, Ludriksson K, Agnon C (1980) Genital tuberculosis in women. Am J Obstet Gynecol 138:933

20. Fox H, Buckely CH (1981) Histopathological study of endometrium in infertility cases. Recent advances in histopathology

21. Hughes EC (1945) Relationship of glycogen to problems of sterility and ovular life. Am J Obstet Gynecol 49:10-18

22. Rozati R, Roopa S, Naga Rajeshwari C (2006) Evaluation of women with infertility and genital tuberculosis. J Obstet Gynecol India 56: 423-426

23. Asha B, Hansali N, Manila K, Priti S, Dhawal B (2011) J Obstet Gynecol India 61(3):301-306 